Research Article

\title{
Cross-sectional descriptive study of lifestyle patterns of undergraduate medical students in a metropolitan Medical College
}

\author{
Pruthvi H. Patel, Aniruddha A. Malgaonkar*, Sundaram Kartikeyan
}

Department of Community Medicine, Rajiv Gandhi Medical College, Kalwa, Thane - 400605, Maharashtra, India

Received: 08 August 2016

Accepted: 06 September 2016

\section{*Correspondence:}

Dr. Aniruddha A. Malgaonkar,

E-mail: andydr@rediffmail.com

Copyright: (c) the author(s), publisher and licensee Medip Academy. This is an open-access article distributed under the terms of the Creative Commons Attribution Non-Commercial License, which permits unrestricted non-commercial use, distribution, and reproduction in any medium, provided the original work is properly cited.

\section{ABSTRACT}

Background: Studies report physical inactivity, mental stress, tobacco and alcohol use and unhealthy and untimely dietary habits in a sizeable proportion of medical students. This complete-enumeration, descriptive study was conducted in a metropolitan teaching hospital to assess the dietary habits and lifestyle of undergraduate medical students for designing specific health interventions based on the results of the study.

Methods: After obtaining permissions and written informed consent, the participants were interviewed and also asked to self-rate their stress on a scale of zero to nine. Their heights and weights were recorded.

Results: Lifestyle patterns of male $(n=78)$ and female $(n=94)$ respondents included vegetarianism (27.9\%), skipping breakfast $(13.95 \%)$, consuming four or more meals daily $(61.63 \%)$, snacking between meals $(43.02 \%)$, consumption of caffeinated soft drinks $(38.37 \%)$ and tea/coffee $(84.31 \%)$, sleeping for 6-8 hours daily $(80.81 \%)$ with no gender difference in minimum and maximum sleep hours, lack of physical exercise $(65.12 \%)$ or outdoor recreation $(25.58 \%)$ and dominance of internet, television and computers as recreational tools. $60.46 \%$ studied only before exams. None were tobacco or alcohol habitual. $94.19 \%$ students had regular bowel habits and $68.03 \%$ had never suffered from constipation. Self-rated stress was often study-related and only $6.98 \%$ sought medical opinion for stress. Menstrual cycles in female respondents were irregular (14.89\%), with mood changes $(69.15 \%)$ and adversely affected studies $(19.15 \%)$.

Conclusions: The same recreational tools utilized by the respondents can be deployed for educational interventions to modify the identified risk factors in this study.

Keywords: Lifestyle, Medical students, Diet, Stress

\section{INTRODUCTION}

A widespread perception is that medical students, being future physicians, would have healthier lifestyle and dietary habits as compared to their non-medical counterparts.

Studies have reported high prevalence of physical inactivity, mental stress, tobacco and alcohol use among a considerable proportion of medical students. ${ }^{1-5}$ Many medical students in the United Arab Emirates were aware that their diet was unhealthy. ${ }^{6}$ A majority of female
Chinese University students with low body mass index (BMI) indicated a desire to be thin. ${ }^{7}$ Unhealthy habits introduced during young adulthood usually persist in later life and can increase the risk of several chronic diseases. $^{8,9}$ In a survey in a college population, $36 \%$ respondents revealed that time constraints posed a barrier to adoption of healthy practices. ${ }^{8}$ Initiation of unhealthy behaviours in medical students may be ascribed to peer pressure, mental stress, performance anxiety in a competitive academic environment, socio-cultural adjustment problems, inadequate parental supervision, home sickness after leaving their parental homes, and 
greater financial autonomy as compared to their earlier years.

Since their behaviours are amenable to change, studentship is particularly the right period for educational interventions regarding the importance of developing and maintaining healthy behaviours. ${ }^{10}$

Interventions to improve the health and lifestyle of medical students would facilitate reduction of their own health risks and would also benefit their future patients because physicians with healthy lifestyles are more likely to inculcate similar behaviours in their patients. ${ }^{11}$

The present study was carried to assess the dietary habits and lifestyle of undergraduate medical students in a metropolitan teaching hospital in order to design specific interventions based on the results of the study.

\section{METHODS}

This complete-enumeration, descriptive study was conducted in 2015 in a municipal medical college in Kalwa, Thane (located about 30 kilometres from Mumbai city). After obtaining permissions from the Institutional Ethics Committee (IEC) and institutional authorities, the purpose of the study was explained to students enrolled for the Bachelor of Medicine, Bachelor of Surgery (MBBS) course and written informed consent was taken from those willing to participate in the study.

The respondents were interviewed by a single observer using a pre-tested semi-structured proforma and were asked to grade their own stress on a scale of zero to nine. For the purpose of this study, a self-rated scale of zero was considered as "stress-free", 1-3 as "low-level stress", 4-7 as "moderate-level stress" and 8-9 as "high-level stress".

Height was measured in centimetres with a scale marked on a vertical wall, with the subject standing in erect position without shoes or headgear with head in Frankfort plane, feet together, heels, buttocks and upper part of the back touching the scale. ${ }^{12}$

Body weight was measured in kilograms using a precalibrated digital weighing scale (OMRON Healthcare India Pvt. Ltd., Gurgaon, Haryana) with the subject standing evenly on both feet, wearing normal indoor clothing but without footwear and before lunch. ${ }^{12}$

Body Mass Index (BMI) was calculated using the formula - weight (in kilograms) / [height (in metres) $]^{2}$. BMI value of 25 or more was taken as the cut-off point for defining overweight in both males and females. ${ }^{13}$

Categorical data were presented as frequencies and percentages and continuous data as Mean and Standard deviation (SD). Significance of difference in parameters was calculated by Karl Pearson's Chi Square test at 95\% confidence interval $(\mathrm{p}<0.05)$.

EpiInfo Version 7.0 (public domain software package from the Centers for Disease Control and Prevention, Atlanta, GA, USA) was used for calculating the Chi Square test (with Yates' correction where applicable) and Odds Ratio (OR).

\section{RESULTS}

A total of 172 students (94 females and 78 males) participated in the study. The median age was 20 years with a SD of 1.49 years (males) and 20 years with a SD of 1.45 years (female). The median, first quartile $\left(\mathrm{Q}_{1}\right)$, and third quartile $\left(\mathrm{Q}_{3}\right)$ were the same for males and females but males had a wider age-range (Figure 1).

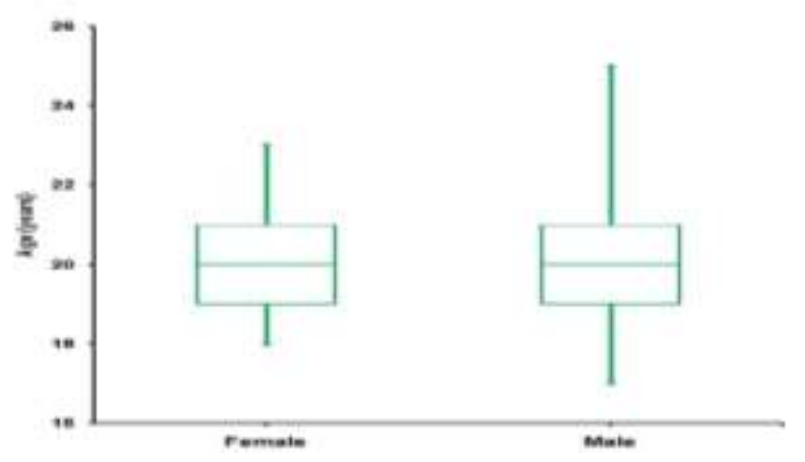

Figure 1: Box plot depicting age-distribution.

\section{Anthropometry}

The median height was $165 \mathrm{~cm}$ with a SD of $10.01 \mathrm{~cm}$; median weight was $58 \mathrm{~kg}$ with a SD of $11.21 \mathrm{~kg}$. The median heights of males and females were $167 \mathrm{~cm}$ and $152.65 \mathrm{~cm}$, respectively (Figure 2). The minimum weight was the same in both sexes but $\mathrm{Q}_{1}$, Median, $\mathrm{Q}_{3}$ and maximum weight was more in males (Figure 3 ).

The median BMI was 21.09 with a SD of 4.6. The minimum BMI of males was lower than that of females but $\mathrm{Q}_{1}$, Median, $\mathrm{Q}_{3}$ and maximum $\mathrm{BMI}$ was higher in males (Figure 4).

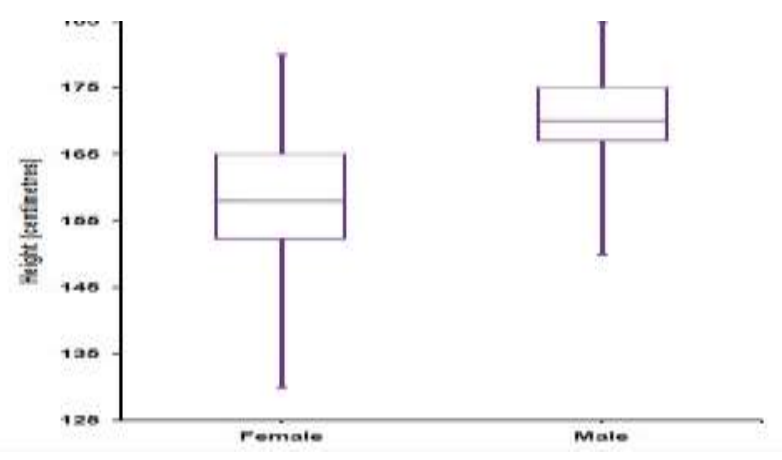

Figure 2: Box plot showing heights of respondents. 


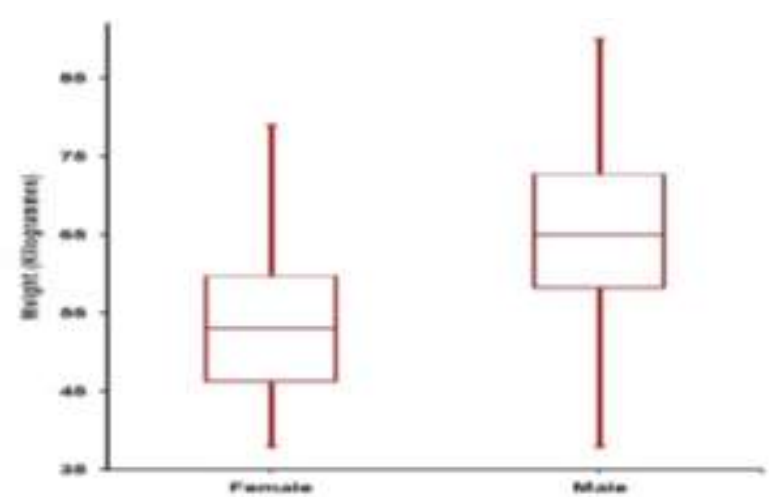

Figure 3: Box plot showing weights of respondents.

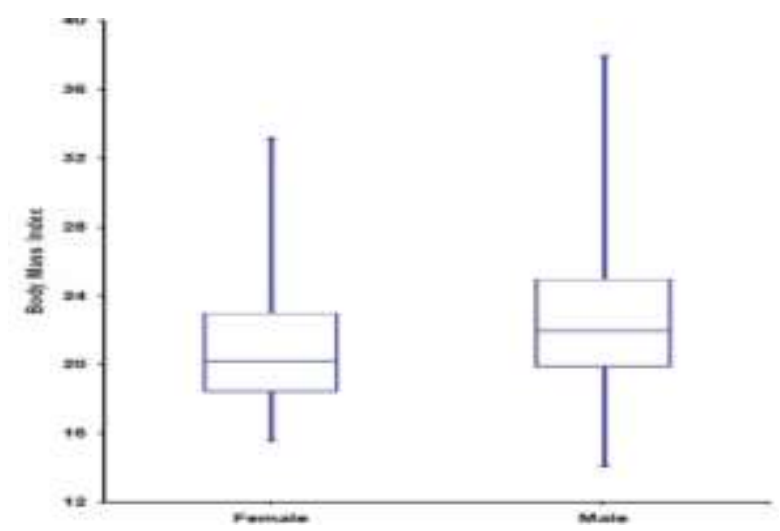

Figure 4: Box plot - body mass index.

Table 1: Pattern of food and beverage consumption.

\begin{tabular}{|c|c|c|c|c|c|c|c|}
\hline \multirow[t]{2}{*}{ Parameter } & & \multicolumn{2}{|c|}{ Females $(n=94)$} & \multicolumn{2}{|c|}{ Males $(\mathrm{n}=78)$} & \multirow{2}{*}{$\begin{array}{l}\mathrm{Chi}^{2} \\
\text { Value }\end{array}$} & \multirow[t]{2}{*}{ p value } \\
\hline & & No. & $\%$ & No. & $\%$ & & \\
\hline \multirow[t]{4}{*}{ Type of diet } & Regularly non-veg & 24 & 13.95 & 17 & 9.88 & \multirow[t]{4}{*}{6.39} & \multirow[t]{4}{*}{0.093} \\
\hline & Vegetarian & 32 & 18.60 & 16 & 9.30 & & \\
\hline & Vegetarian + eggs & 11 & 6.40 & 17 & 9.88 & & \\
\hline & Rarely non-veg & 27 & 15.70 & 28 & 16.28 & & \\
\hline \multirow[t]{5}{*}{ Meals per day } & Breakfast & 82 & 47.67 & 66 & 38.37 & \multirow[t]{5}{*}{0.332} & \multirow[t]{5}{*}{0.961} \\
\hline & Mid-Morning & 20 & 11.63 & 14 & 8.14 & & \\
\hline & Lunch & 88 & 51.16 & 73 & 42.44 & & \\
\hline & Evening & 68 & 39.53 & 55 & 31.98 & & \\
\hline & Dinner & 92 & 53.49 & 74 & 43.02 & & \\
\hline \multirow{3}{*}{$\begin{array}{l}\text { Eating Between } \\
\text { Meals }\end{array}$} & Daily & 9 & 5.23 & 16 & 9.30 & \multirow[t]{3}{*}{4.648} & \multirow[t]{3}{*}{0.098} \\
\hline & Sometimes & 70 & 40.7 & 48 & 27.91 & & \\
\hline & Never & 15 & 8.72 & 14 & 8.14 & & \\
\hline \multirow{3}{*}{$\begin{array}{l}\text { Consumption: } \\
\text { Caffeinated soft } \\
\text { drinks }\end{array}$} & Daily & 2 & 1.16 & 5 & 2.91 & \multirow[t]{3}{*}{4.214} & \multirow[t]{3}{*}{0.122} \\
\hline & Sometimes & 71 & 41.28 & 63 & 36.62 & & \\
\hline & Never & 21 & 12.21 & 10 & 5.81 & & \\
\hline \multirow{3}{*}{$\begin{array}{l}\text { Consumption: } \\
\text { Coffee/Tea }\end{array}$} & Daily & 0 & 0.00 & 4 & 2.33 & \multirow[t]{3}{*}{5.44} & \multirow[t]{3}{*}{0.066} \\
\hline & Never & 3 & 1.74 & 4 & 2.33 & & \\
\hline & Sometimes & 91 & 52.91 & 70 & 40.7 & & \\
\hline
\end{tabular}

Table 2: Dietary pattern, physical activities and body mass index (BMI).

\begin{tabular}{|llllll|}
\hline Parameter & $\mathbf{B M I}:<\mathbf{2 5}(\mathbf{n}=\mathbf{1 4 2})$ & $\mathbf{B M I}: \mathbf{2 5 +}(\mathbf{n}=\mathbf{3 0})$ & $\mathbf{C h}^{\mathbf{2}}$ Value & $\mathbf{p}$ value & Odds Ratio \\
\hline Three or more meals per day & 52 & 40 & 10.74 & 0.001 & 0.325 \\
\hline Never eating between meals & 21 & 8 & 2.492 & 0.114 & 0.4773 \\
\hline Frequently eating between meals & 17 & 8 & 4.305 & $0.038 \#$ & 0.374 \\
\hline Only vegetarian & 39 & 9 & 0.079 & 0.778 & 0.883 \\
\hline Regular non-vegetarian & 34 & 7 & 0.005 & 0.943 & 0.967 \\
\hline Gymnasium workouts - Never & 98 & 14 & 5.445 & $0.0196 \#$ & 2.545 \\
\hline Gymnasium workouts - frequent & 9 & 6 & 5.807 & $0.0159 \#$ & 0.2707 \\
\hline No outdoor activity & 32 & 12 & 3.968 & $0.046 \#$ & 0.436 \\
\hline Frequent outdoor activity & 9 & 2 & 0.118 & 0.73 & 0.947 \\
\hline
\end{tabular}

\# statistically significant.

\section{Food habits}

$27.9 \%$ were vegetarians while $23.83 \%$ regularly consumed non-vegetarian diet. $61.63 \%$ had four or more meals per day; $43.02 \%$ ate between meals on a daily or frequent basis. Frequency of regular breakfast, lunch and dinner was $86.05 \%, 93.6 \%$, and $96.51 \%$, respectively. Regular consumption of caffeinated soft drinks and tea or 
coffee was reported by $38.37 \%$ and $84.31 \%$, respectively (Tables 1-3).

\section{Sleep pattern}

$80.81 \%$ students slept for 6-8 hours per day. $55.82 \%$ slept once a day without waking in between. Only $17.45 \%$ students had regular daytime naps (Table 4). There was no gender difference in minimum and maximum sleep hours.

There was no difference between [minimum \& $\mathrm{Q}_{1}$ ] and [median \& $\left.\mathrm{Q}_{3}\right]$ in males and no difference in $\left[\mathrm{Q}_{1} \&\right.$ median] in females indicating fixed similar hours of sleep in many students (Figure 5).

Table 3: Gender-wise dietary pattern and physical activities.

\begin{tabular}{|llllll|}
\hline Parameter & Females $(\mathbf{n}=\mathbf{9 4})$ & Males $(\mathbf{n = 7 8})$ & Chi $^{2}$ Value & p value & Odds Ratio \\
\hline Three or more meals per day & 33 & 33 & 0.935 & 0.334 & 0.737 \\
\hline Never eating between meals & 15 & 14 & 0.127 & 0.728 & 0.868 \\
\hline Frequently eating between meals & 9 & 16 & 4.105 & $0.043 \#$ & 0.410 \\
\hline Only vegetarian & 32 & 16 & 3.878 & $0.049 \#$ & 2.00 \\
\hline Regular non-vegetarian & 24 & 17 & 0.327 & 0.567 & 1.23 \\
\hline Gymnasium workouts - never & 72 & 40 & 46.456 & $0.0000 \#$ & 16.2 \\
\hline Gymnasium workouts - frequent & 3 & 12 & 3.213 & 0.073 & 0.273 \\
\hline No outdoor activity & 31 & 18 & 8.874 & $0.003 \#$ & 2.78 \\
\hline Frequent outdoor activity & 0 & 6 & 3.437 & 0.063 & $\ldots$ \\
\hline
\end{tabular}

\# statistically significant.

Table 4: Sleep patterns.

\begin{tabular}{|c|c|c|c|c|c|c|c|}
\hline \multirow[t]{2}{*}{ Sleep patterns } & & \multicolumn{2}{|c|}{ Females $(n=94)$} & \multicolumn{2}{|c|}{ Males $(\mathrm{n}=78)$} & \multirow[t]{2}{*}{$\mathrm{Chi}^{2}$ Value } & \multirow[t]{2}{*}{ p value } \\
\hline & & No. & $\%$ & No. & $\%$ & & \\
\hline \multirow[t]{2}{*}{ Duration of sleep (hours) } & $6-8$ & 80 & 46.51 & 59 & 34.30 & \multirow[t]{2}{*}{2.46} & \multirow[t]{2}{*}{0.117} \\
\hline & $9-12$ & 14 & 8.14 & 19 & 11.05 & & \\
\hline \multirow[t]{3}{*}{ Wakes up during sleep } & No & 49 & 28.49 & 47 & 27.33 & \multirow[t]{3}{*}{1.80} & \multirow[t]{3}{*}{0.406} \\
\hline & Sometimes & 33 & 19.19 & 20 & 11.63 & & \\
\hline & Yes & 12 & 6.98 & 11 & 6.40 & & \\
\hline \multirow[t]{3}{*}{ Daytime naps } & Daily & 12 & 6.98 & 18 & 10.47 & \multirow[t]{3}{*}{3.331} & \multirow[t]{3}{*}{0.189} \\
\hline & Sometimes & 66 & 38.38 & 50 & 29.07 & & \\
\hline & Never & 16 & 9.30 & 10 & 5.81 & & \\
\hline
\end{tabular}

Table 5: Physical activity.

\begin{tabular}{|c|c|c|c|c|c|c|c|}
\hline & & \multicolumn{2}{|c|}{ Females $(n=94)$} & \multicolumn{2}{|c|}{ Males $(n=78)$} & \multirow[t]{2}{*}{ Chi $^{2}$ Value } & \multirow[t]{2}{*}{ p value } \\
\hline & & No. & $\%$ & No. & $\%$ & & \\
\hline \multirow[t]{2}{*}{ Physical activity } & Irregular & 38 & 22.09 & 33 & 19.19 & \multirow[t]{2}{*}{0.062} & \multirow[t]{2}{*}{0.802} \\
\hline & Regular & 56 & 32.56 & 45 & 26.16 & & \\
\hline \multirow{3}{*}{$\begin{array}{l}\text { Frequency of outdoor } \\
\text { games }\end{array}$} & Daily & 4 & 2.33 & 7 & 4.07 & \multirow[t]{3}{*}{1.907} & \multirow[t]{3}{*}{0.385} \\
\hline & Sometimes & 67 & 38.95 & 50 & 29.07 & & \\
\hline & Never & 23 & 13.37 & 21 & 12.21 & & \\
\hline \multirow{3}{*}{$\begin{array}{l}\text { Frequency of indoor } \\
\text { games }\end{array}$} & Daily & 0 & 0.00 & 6 & 3.49 & \multirow[t]{3}{*}{8.728} & \multirow[t]{3}{*}{$0.013 \#$} \\
\hline & Sometimes & 63 & 36.63 & 54 & 31.4 & & \\
\hline & Never & 31 & 18.02 & 18 & 10.47 & & \\
\hline \multirow{3}{*}{$\begin{array}{l}\text { Frequency of going to } \\
\text { gym }\end{array}$} & Daily & 3 & 1.74 & 12 & 6.98 & \multirow[t]{3}{*}{14.266} & \multirow[t]{3}{*}{0.0008 \# } \\
\hline & Sometimes & 19 & 11.04 & 26 & 15.12 & & \\
\hline & Never & 72 & 41.86 & 40 & 23.26 & & \\
\hline
\end{tabular}

\# statistically significant.

\section{Physical activity}

$8.72 \%$ exercised in the gymnasium daily while $65.12 \%$ students never did so (Tables 2-5).

\section{Recreation}

$58.72 \%$ had a regular recreation pattern while $25.58 \%$ never had any outdoor recreation. $41.24 \%$ regularly 
watched television and $88.96 \%$ regularly surfed the internet (Table 6).

\section{Study pattern}

$2.90 \%$ students had regular study pattern, $36.62 \%$ studied irregularly while $60.46 \%$ studied only before exams.
$48.25 \%$ preferred studying at night and $88.38 \%$ preferred a sitting posture while studying. $84.30 \%$ preferred to study alone (Table 7).

\section{Addictions}

None of the respondents in this study were habitual users of tobacco or alcohol.

Table 6: Pattern of recreation.

\begin{tabular}{|c|c|c|c|c|c|c|c|}
\hline \multicolumn{2}{|c|}{ Pattern of recreation } & \multicolumn{2}{|c|}{ Females $(n=94)$} & \multicolumn{2}{|c|}{ Males $(\mathbf{n}=78)$} & \multirow{2}{*}{$\begin{array}{l}\text { Chi }^{2} \\
\text { Value }\end{array}$} & \multirow[t]{2}{*}{ p value } \\
\hline & & No. & $\%$ & No. & $\%$ & & \\
\hline \multirow[t]{3}{*}{ Television } & Daily & 42 & 24.42 & 29 & 16.86 & \multirow[t]{3}{*}{1.162} & \multirow[t]{3}{*}{0.559} \\
\hline & Sometimes & 37 & 21.52 & 33 & 19.18 & & \\
\hline & Never & 15 & 8.72 & 16 & 9.30 & & \\
\hline \multirow[t]{3}{*}{ Computer } & Daily & 32 & 18.60 & 22 & 12.79 & \multirow[t]{3}{*}{1.253} & \multirow[t]{3}{*}{0.534} \\
\hline & Sometimes & 46 & 26.75 & 38 & 22.1 & & \\
\hline & Never & 16 & 9.30 & 18 & 10.47 & & \\
\hline \multirow[t]{3}{*}{ Internet } & Daily & 83 & 48.26 & 70 & 40.70 & \multirow[t]{3}{*}{1.518} & \multirow[t]{3}{*}{0.468} \\
\hline & Sometimes & 11 & 6.39 & 7 & 4.07 & & \\
\hline & Never & 0 & 0.00 & 1 & 0.58 & & \\
\hline
\end{tabular}

Table 7: Study pattern.

\begin{tabular}{|c|c|c|c|c|c|c|c|}
\hline \multicolumn{2}{|c|}{ Pattern of studying } & \multicolumn{2}{|c|}{ Females $(n=94)$} & \multicolumn{2}{|c|}{ Males $(n=78)$} & \multirow[t]{2}{*}{ Chi $^{2}$ Value } & \multirow[t]{2}{*}{ p value } \\
\hline & & No. & $\%$ & No. & $\%$ & & \\
\hline \multirow[t]{3}{*}{ Regularity } & Regular & 3 & 1.74 & 2 & 1.16 & \multirow[t]{3}{*}{2.30} & \multirow[t]{3}{*}{0.316} \\
\hline & Irregular & 39 & 22.67 & 24 & 13.95 & & \\
\hline & Only during exams & 52 & 30.23 & 52 & 30.23 & & \\
\hline \multirow[t]{4}{*}{ Time of Study } & Morning & 24 & 13.95 & 7 & 4.07 & \multirow[t]{4}{*}{9.302} & \multirow[t]{4}{*}{$0.025 \#$} \\
\hline & Evening & 19 & 11.05 & 14 & 8.14 & & \\
\hline & Night & 38 & 22.09 & 45 & 26.16 & & \\
\hline & Others & 13 & 7.56 & 12 & 6.98 & & \\
\hline \multirow{3}{*}{$\begin{array}{l}\text { Posture while } \\
\text { studying }\end{array}$} & Sitting & 83 & 48.26 & 69 & 40.12 & \multirow[t]{3}{*}{0.023} & \multirow[t]{3}{*}{0.988} \\
\hline & Sleeping & 10 & 5.81 & 8 & 4.65 & & \\
\hline & Walking & 1 & 0.58 & 1 & 0.58 & & \\
\hline \multirow[t]{2}{*}{ Study Company } & Alone & 81 & 47.09 & 64 & 37.21 & \multirow[t]{2}{*}{0.546} & \multirow[t]{2}{*}{0.46} \\
\hline & With Others & 13 & 7.56 & 14 & 8.14 & & \\
\hline
\end{tabular}

\# statistically significant

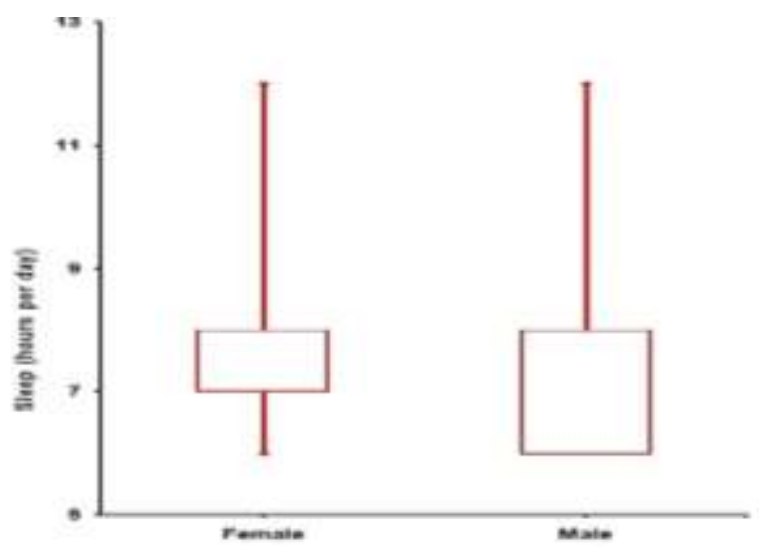

Figure 5: Box plot - daily hours of sleep.

\section{Bowel habits}

94.19\% students had regular bowel habits and $68.03 \%$ had never suffered from constipation. $3.19 \%$ of the females and $8.97 \%$ of the males had irregular bowel habits.

$1.06 \%$ of females and $2.56 \%$ of males often had constipation.

\section{Stress}

The frequency of moderate and high degree of self-rated stress was $56.39 \%$ and $12.80 \%$, respectively. The 
reported causes of stress were studies $(93.02 \%)$ and nonacademic reasons $(6.98 \%)$.

$33.72 \%$ responded that stress adversely affected their studies. For $51.74 \%$ students, exercise was a stressbuster. $6.98 \%$ had taken medical opinion for stress management (Table 8).

\section{Menstrual pattern}

Female students reported irregular menses (14.89\%), bleeding for more than five days $(21.28 \%)$, heavy bleeding $(14.89 \%)$, passage of clots $(52.13 \%)$, mood changes $(69.15 \%)$ and adverse effect of menstrual problems on their studies (19.15\%).

Table 8: Stress.

\begin{tabular}{|c|c|c|c|c|c|c|c|}
\hline \multirow[t]{2}{*}{ Parameters } & & \multicolumn{2}{|c|}{ Females $(n=94)$} & \multicolumn{2}{|c|}{ Males $(n=78)$} & \multirow{2}{*}{$\begin{array}{l}\mathrm{Chi}^{2} \\
\text { Value }\end{array}$} & \multirow[t]{2}{*}{ p value } \\
\hline & & No. & $\%$ & No. & $\%$ & & \\
\hline \multirow[t]{4}{*}{ Stress levels } & 0 (“stress-free”) & 5 & 2.91 & 6 & 3.49 & \multirow[t]{4}{*}{3.56} & \multirow[t]{4}{*}{0.312} \\
\hline & 1-3 ("low") & 19 & 11.05 & 23 & 13.37 & & \\
\hline & 4-7 (“moderate") & 59 & 34.30 & 38 & 22.09 & & \\
\hline & 8-9 (“high") & 11 & 6.40 & 11 & 6.40 & & \\
\hline \multirow[t]{2}{*}{ Reasons for stress } & Studies & 89 & 51.74 & 71 & 41.28 & \multirow[t]{2}{*}{0.877} & \multirow[t]{2}{*}{0.348} \\
\hline & Others & 5 & 2.91 & 7 & 4.07 & & \\
\hline \multirow{3}{*}{$\begin{array}{l}\text { Stress affecting } \\
\text { studies }\end{array}$} & Never & 13 & 7.56 & 20 & 11.63 & \multirow[t]{3}{*}{4.22} & \multirow[t]{3}{*}{0.121} \\
\hline & Sometimes & 49 & 28.49 & 32 & 18.60 & & \\
\hline & Yes & 32 & 18.60 & 26 & 15.12 & & \\
\hline \multirow{4}{*}{$\begin{array}{l}\text { Methods for relieving } \\
\text { stress }\end{array}$} & Exercise & 52 & 30.23 & 37 & 21.51 & \multirow[t]{4}{*}{3.707} & \multirow[t]{4}{*}{0.294} \\
\hline & Family, friends & 16 & 9.30 & 9 & 5.23 & & \\
\hline & Sleep & 13 & 7.56 & 17 & 9.88 & & \\
\hline & Others & 13 & 7.55 & 15 & 8.72 & & \\
\hline \multirow[t]{2}{*}{ Medical opinion } & No & 87 & 50.58 & 73 & 42.44 & \multirow[t]{2}{*}{0.07} & \multirow[t]{2}{*}{0.79} \\
\hline & Yes & 7 & 4.07 & 5 & 2.91 & & \\
\hline
\end{tabular}

\section{DISCUSSION}

Of the total 172 respondents, all were aged between 17 and 25 years. In a longitudinal study among Turkish university nursing students, lifestyle habits were found to change from the beginning to the end of nursing training. ${ }^{14}$

\section{Anthropometry}

$17.44 \%$ had BMI of 25 or more and $4.07 \%$ had BMI more than 30 . Students residing outside the campus were more likely to be obese as compared to their campusresiding counterparts. ${ }^{15}$ In a Greece-based study, $32.1 \%$ of male and $8.4 \%$ of female medical students were overweight. $14.8 \%$ and $5.2 \%$ Malaysian medical students were found to be overweight and obese, respectively. ${ }^{16,17}$

\section{Food habits}

$13.95 \%$ skipped breakfast while $19.77 \%$ students had a mid-morning snack. The difference between obesity rate amongst vegetarian students and others was insignificant $(p=0.728)$ and that amongst non-vegetarian students and others was also insignificant $(\mathrm{p}=0.943)$. However, there was significant difference $(\mathrm{p}=0.001)$ in obesity rate amongst those eating three or more meals per day and those who did not. Similarly, the difference in obesity rate amongst those always eating between regular meals and those who did not was also significant $(\mathrm{p}=0.038)$. In the Goteberg adolescent study, irregular breakfast habit was found to be related to smoking and a lower micronutrient intake. ${ }^{18}$

Healthy eating was more frequent among female Greek students but they had less physical activity as compared to their male counterparts. ${ }^{19}$ Swedish female university students were found to have healthier habits despite being more prone to stress, while their male counterparts were found to have a higher prevalence of obesity, physical inactivity and alcohol consumption. ${ }^{20}$

\section{Sleep pattern}

In this study, almost all respondents slept for more than 6 hours per day. A Japanese study reported significant gender differences in sleep pattern with an evening preponderance with shortened sleep duration during school days. $^{21}$

\section{Physical activity}

The obesity rate was significantly higher $(\mathrm{p}=0.0196)$ among students who never exercised, as compared to their physically active counterparts. The frequency of going to the gymnasium was significantly higher $(p=0.0008)$ amongst males. The obesity rate amongst 
those regularly visiting the gymnasium was significantly lower $(\mathrm{p}=0.015)$ than that in the others.

In a Korean study, only $33.6 \%$ of the student's exercised daily. ${ }^{15}$ Physical activity showed a significant association with healthy eating patterns suggesting that healthful behaviours occurred together. ${ }^{22}$ A Spanish study has recommended nutritional education campaigns that include promotion of physical activity practice are needed to improve the overall health status of this population. $^{23}$

\section{Recreation}

The frequency of playing indoor games was significantly higher $(p=0.013)$ amongst males. Females with no outdoor recreation significantly outnumbered their male counterparts $(p=0.003)$. In a Mangalore-based study, majority of the participants were introduced to junk food by television commercials and only $18.02 \%$ of the students never watched television. ${ }^{24}$

\section{Study pattern}

Most respondents $(95.34 \%)$ had fixed study timings but there was a significant gender difference $(p=0.025)$ in the study pattern. A Japanese study reported that morning study preference was associated with higher sleep drive and better sleep-wake parameters and lifestyle habits; while evening study preference was associated with longer sleep latency, curtailed sleep duration and spells of daytime sleepiness. $^{21}$

\section{Addictions}

In the present study, none of the respondents were habituated to tobacco or alcohol. Ten percent of surveyed American college-going women reportedly smoked to control their weight. ${ }^{22}$ Smokers also had higher alcohol consumption. In a study among Spanish university students, one-third admitted to using illegal substances. ${ }^{25}$ One-third of British medical students consumed alcohol above safe limits and $65 \%$ of them were aware of the dangers. ${ }^{26}$ The rate of binge drinking was reported to be $32 \%$ among German medical students. ${ }^{27}$

\section{Bowel habits}

In the present study, only $2.91 \%$ students often suffered from constipation while $5.81 \%$ students had irregular bowel habits. However, a Korean study reported that $58.4 \%$ students had irregular bowel movements. ${ }^{28}$

\section{Stress}

Only $6.4 \%$ students responded that they were stress-free and majority responded that studies were the cause of stress which, in turn, adversely affected studies. An Australian study has reported an overall enhancement in wellbeing of medical students during the pre-exam period after a de-stressing programme. ${ }^{29}$

\section{Menstrual pattern}

Majority of the female students had regular menstrual pattern with 4-5 days of bleeding and moderate flow but menstruation adversely affected studies. A Saudi Arabian study has attributed low class attendance of female medical students to dysmenorrhoea and premenstrual syndrome. $^{30}$ The limitations are that this study was conducted in only one institution and it was not possible to verify the self-reported data provided by the respondents. The findings of a larger multi-centric study would help in formulating educational and health promotion interventions for undergraduate medical students.

\section{CONCLUSION}

This study reveals presence of various modifiable risk factors, such as obesity, unhealthy diet and irregular eating habits, physical inactivity and stress among the respondents. These risk factors are amenable to educational and health promotion interventions. Since recreation in this study group was dominated by computer, television and the internet, these very tools can be used judiciously to impart educational interventions on lifestyle modification.

\section{Funding: No funding sources}

Conflict of interest: None declared

Ethical approval: The study was approved by the Institutional Ethics Committee

\section{REFERENCES}

1. Troyer D, Ullrich IH, Yeater RA, Hopewell R. Physical activity and condition, dietary habits, and serum lipids in second-year medical students. J Am Coll Nutr. 1990;9:303-7.

2. Iqbal S, Gupta S, Venkatarao E. Stress, anxiety \& depression among medical undergraduate students $\&$ their socio-demographic correlates. Indian J Med Res. 2015;141(3):354-7.

3. Singh VV, Singh Z, Banerjee A, Basannar DR. Determinants of Smoking Habit among Medical Students. MJAFI. 2003;59(3):209-11.

4. Goel N, Khandelwal V, Pandya K, Kotwal A. Alcohol and Tobacco Use Among Undergraduate and Postgraduate Medical Students in India: A Multicentric Cross-sectional Study. Central Asian Journal of Global Health. 2015;4(1).

5. Collier DJ, Beales IL. Drinking among medical students: a questionnaire survey. BMJ. 1989; 299(6690):19-22.

6. Carter AO, Elzubeir M, Abdulrazzaq YM, Revel AD, Townsend A. Health and lifestyle needs assessment of medical students in the United Arab Emirates. Med Teac. 2003;25:492-6. 
7. Sakamaki R, Toyama K, Amamoto R, Liu CJ, Shinfuku N. Nutritional knowledge, food habits and health attitude of Chinese university students-a cross sectional study. Nutr J. 2005;4:4.

8. Webb E, Ashton CH, Kelly P, Kamah F. An update on British medical students' lifestyles. Med Educ. 1998;32:325-31.

9. Cullen KW, Koehly LM, Anderson C, Baranowski $\mathrm{T}$, Prokhorov A, Basen-Engquist K, et al. Gender differences in chronic disease risk behaviours through the transition out of high school. Am J Prev Med. 1999;17(1):1-7.

10. Schnoll R, Zimmerman BJ. Self-regulation training enhances dietary self-efficacy and dietary fibre consumption. Centres for Disease Control and Prevention. Nutrition: School Health Guidelines 2001.

11. Lobelo F, Duperly J, Frank E. Physical activity habits of doctors and medical students influence their counselling practices. $\mathrm{Br} \mathrm{J}$ Sports Med. 2009;43(2):89-92.

12. International Society for the Advancement of Kinanthropometry (ISAK). International Standards for Anthropometric Assessment. Underdale, SA, Australia. 2001:53-5.

13. Bray GA. In defense of a body mass index of 25 as the cut-off point for defining overweight. Obes Res. 1998;6(6):461-2.

14. Alpara SE, Şenturanb L, Karabacaka U, Sabuncub N. Change in the health promoting lifestyle behaviour of Turkish University nursing students from beginning to end of nurse training. Nurse Educ Pract. 2008;8(6):382-8.

15. Chung HJ, Park HW, Choi EJ, Lee JJ. A study of the lifestyle factors related to constipation among food habits of college students in Seoul and Gyunggi. Korean J Community Nutr. 2002;7(5):654-63.

16. Chourdakis M, Tzellos T, Papazisis G, Toulis K, Kouvelas D. Eating habits, health attitudes and obesity indices among medical students in northern Greece. Appetite. 2010;55(3):722-5.

17. Gopalakrishnan S, Ganeshkumar P, Prakash MV, Christopher, Amalraj V. Prevalence of overweight/ obesity among the medical students, Malaysia. Med J Malaysia. 2012;67(4):442-4.

18. Sjöberg A, Hallberg L, Höglund D, Hulthén L. Meal pattern, food choice, nutrient intake and lifestyle factors in The Göteborg Adolescence Study. Eur J Clin Nutr. 2003;57:1569-78.

19. Tirodimos I, Georgouvia I, Savvala TN, Karanika E, Noukari D. Healthy lifestyle habits among Greek university students: differences by sex and faculty of study. East Mediterr Health J. 2009;15(3):722-8.
20. von Bothmer MI, Fridlund B. Gender differences in health habits and in motivation for a healthy lifestyle among Swedish university students. Nurs Health Sci. 2005;7:107-18.

21. Gaina A, Sekinea M, Kanayamaa H, Takashib Y, Hua L, Sengokua K, et al. Morning-Evening Preference: Sleep Pattern Spectrum and Lifestyle Habits among Japanese Junior High School pupils. Chronobiol Int. 2006;23(3):607-21.

22. Hendricks KM, Herbold N, Fung T. Diet and other lifestyle behaviors in young college women. Nutr. Res. 2004;24(12):981-91.

23. Moreno-Gómeza C, Romaguera-Boscha D, TaulerRieraa P, Bennasar-Venya M, Pericas-Beltrana J, Martinez-Andreua S, et al. Clustering of lifestyle factors in Spanish university students: the relationship between smoking, alcohol consumption, physical activity and diet quality. Public Health Nutr. 2012;15(11):2131-9.

24. Joseph N, Nelliyanil M, Rai S, Y P RB, Kotian SM, Ghosh T, Singh M. Fast Food consumption pattern and its association with overweight among high school boys in Mangalore City of Southern India. J Clin Diagn Res. 2015:9(5):LC13-7.

25. Varela-Mato V, Cancela JM, Ayan C, Martin V, Molina A. Lifestyle and Health among Spanish University Students: Differences by Gender and Academic Discipline. Int. J. Environ. Res. Publ. Health. 2012;9(8):2728-41.

26. File SE, Mabbutt PS, Shaffer J. Alcohol consumption and lifestyle in medical students. J Psychopharmacol. 1994;8(1):22-6.

27. Keller S, Maddock JE, Laforge RG, Velicer WF, Basler HD. Binge drinking and health behavior in medical students. Addict Behav. 2007;32(3):50515.

28. Lee JH, O JH. A study on the defecation pattern and lifestyle factors of female high school and college students in Gyeonggi Province. Korean J Community Nutr. 2005;10(1):36-45.

29. Hassed C, de Lisle S, Sullivan G, Pier C. Enhancing the health of medical students: outcomes of an integrated mindfulness and lifestyle program. Adv. Health Sci. Educ. 2009;14(3):387-98.

30. Aref N, Rizwan F, Abbas MM. Frequency of Different Menstrual Disorders among Female Medical Students at Taif Medical College. World J Med Sci. 2015;12(2):109-14.

Cite this article as: Patel $\mathrm{PH}$, Malgaonkar AA, Kartikeyan S. Cross-sectional descriptive study of lifestyle patterns of undergraduate medical students in a metropolitan Medical College. Int J Community Med Public Health 2016;3:2866-73. 